University of Nebraska - Lincoln

DigitalCommons@University of Nebraska - Lincoln

Fumonisin Production in the Maize Pathogen Fusarium verticillioides: Genetic Basis of Naturally Occurring Chemical Variation

\author{
Robert H. Proctor \\ USDA-ARS, proctorh@ncaur.usda.gov \\ Ronald D. Plattner \\ USDA-ARS \\ Anne E. Desjardins \\ USDA-ARS \\ Mark Busman \\ USDA-ARS \\ Robert A. E. Butchko \\ USDA-ARS
}

Follow this and additional works at: https://digitalcommons.unl.edu/usdaarsfacpub

Proctor, Robert H.; Plattner, Ronald D.; Desjardins, Anne E.; Busman, Mark; and Butchko, Robert A. E., "Fumonisin Production in the Maize Pathogen Fusarium verticillioides: Genetic Basis of Naturally Occurring Chemical Variation" (2006). Publications from USDA-ARS / UNL Faculty. 1570.

https://digitalcommons.unl.edu/usdaarsfacpub/1570

This Article is brought to you for free and open access by the U.S. Department of Agriculture: Agricultural Research Service, Lincoln, Nebraska at DigitalCommons@University of Nebraska - Lincoln. It has been accepted for inclusion in Publications from USDA-ARS / UNL Faculty by an authorized administrator of DigitalCommons@University of Nebraska - Lincoln. 


\title{
Fumonisin Production in the Maize Pathogen Fusarium verticillioides: Genetic Basis of Naturally Occurring Chemical Variation
}

\author{
Robert H. Proctor, * Ronald D. Plattner, Anne E. Desjardins, \\ Mark Busman, and Robert A. E. ButchKo \\ National Center for Agricultural Utilization Research, Agricultural Research Service, \\ U.S. Department of Agriculture, 1815 North University Street, Peoria, Illinois 61604
}

\begin{abstract}
Fumonisins are polyketide-derived mycotoxins produced by the maize pathogen Fusarium verticillioides. Previous analyses identified naturally occurring variants of the fungus that are deficient in fumonisin C-10 hydroxylation or that do not produce any fumonisins. In the current study, gene deletion and genetic complementation analyses localized the C-10 hydroxylation deficiency to a cytochrome P450 monooxygenase gene in the fumonisin biosynthetic gene (FUM) cluster. Sequence analysis indicated that the hydroxylation deficiency resulted from a single nucleotide insertion that caused a frame shift in the coding region of the gene. Genetic complementation localized the fumonisinnonproduction phenotype to the polyketide synthase gene in the FUM cluster, and sequence analysis indicated that the nonproduction phenotype resulted from a nucleotide substitution, which introduced a premature stop codon in the coding region. These results provide the first direct evidence that altered fumonisin production phenotypes of naturally occurring $F$. verticillioides variants can result from single point mutations in the FUM cluster.
\end{abstract}

KEYWORDS: Fusarium verticillioides; fumonisins; fumonisin biosynthesis; biosynthetic gene; polyketide synthase; cytochrome P450 monooxygenase

\section{INTRODUCTION}

Fumonisins are polyketide mycotoxins that can accumulate in maize and can cause animal health problems, including cancer and neural tube defects in laboratory rodents $(1,2)$. Consumption of fumonisin-contaminated maize has also been epidemiologically correlated with human esophageal cancer and neural tube defects in some regions of the world $(3,4)$. Fumonisins are produced by several relatively closely related species of the genus Fusarium $(5,6)$. Of these fungal species, $F$. verticillioides (sexual state Gibberella moniliformis) has received the most attention because of its widespread occurrence on maize and its ability to cause maize ear and stalk rot (7). F. verticillioides typically produces fumonisins $\mathrm{B}_{1}, \mathrm{~B}_{2}, \mathrm{~B}_{3}$, and $\mathrm{B}_{4}\left(\mathrm{FB}_{1}, \mathrm{FB}_{2}\right.$, $\mathrm{FB}_{3}$, and $\mathrm{FB}_{4}$, respectively). These compounds share a linear 20-carbon backbone with an amine at carbon atom 2 (C-2) and tricarboxylic acid moieties esterified to $\mathrm{C}-14$ and $\mathrm{C}-15$ (8). The compounds differ from one another by the presence or absence of hydroxyl functions at $\mathrm{C}-5$ and $\mathrm{C}-10$ (Figure 1). $\mathrm{FB}_{1}$ has both hydroxyls, $\mathrm{FB}_{2}$ lacks the $\mathrm{C}-10$ hydroxyl, $\mathrm{FB}_{3}$ lacks the $\mathrm{C}-5$ hydroxyl, and $\mathrm{FB}_{4}$ lacks both the $\mathrm{C}-5$ and $\mathrm{C}-10$ hydroxyls. In general, $\mathrm{FB}_{1}$ is the most abundant fumonisin $(\sim 70 \%)$ in naturally contaminated maize and in cultures of most field

* Corresponding author [telephone (309) 681-6380; fax (309) 681-6686; e-mail proctorh@ncaur.usda.gov].

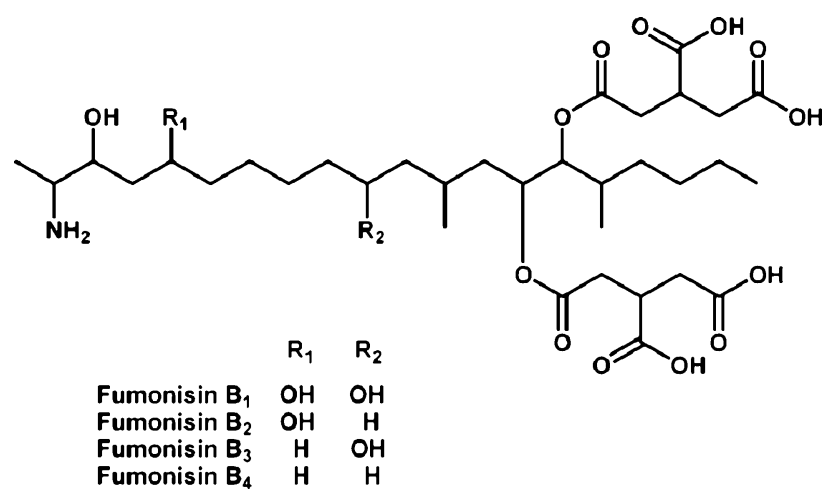

Figure 1. Chemical structures of fumonisins $B_{1}, B_{2}, B_{3}$, and $B_{4}$.

isolates of $F$. verticillioides, whereas $\mathrm{FB}_{2}, \mathrm{FB}_{3}$, and $\mathrm{FB}_{4}$ are less abundant (9).

In surveys of $F$. verticillioides aimed at examining the diversity of fumonisin chemotypes and their potential in the biological control of fumonisin contamination, researchers have identified naturally occurring variants of the fungus with relatively rare chemotypes $(10,11)$. One variant isolated from maize from Nepal produced no fumonisins; another variant isolated from maize from South Carolina was deficient in C-5 hydroxylation and, as a result, produced only $\mathrm{FB}_{3}$ and $\mathrm{FB}_{4}$; and a third variant, also isolated from maize from South Carolina, 
Table 1. F. verticillioides Strains Used in the Study

\begin{tabular}{|c|c|c|c|c|c|}
\hline strain & fumonisin production ${ }^{a}$ & genotype $^{a}$ & strain origin & parent strains & ref \\
\hline M-3125 & $\mathrm{FB}_{1}, \mathrm{FB}_{2}, \mathrm{FB}_{3}, \mathrm{FB}_{4}$ & Fum1-1, Fum2-1 & field isolate & $N / A^{b}$ & 35 \\
\hline ISU-93-152 & none & $\mathrm{nd}^{b}$ & field isolate & $\mathrm{N} / \mathrm{A}$ & 31 \\
\hline $109-R-14$ & $\mathrm{FB}_{2}, \mathrm{FB}_{4}$ & Fum1-1, Fum2-2 & meiotic progeny & $\begin{array}{l}\mathrm{M}-3120^{\circ}(\%) \\
\mathrm{A}-0822^{\mathrm{C}}\left(0^{*}\right)\end{array}$ & 13 \\
\hline $982-R-50$ & $\mathrm{FB}_{2}, \mathrm{FB}_{4}$ & Fum1-1, Fum2-2 & meiotic progeny & $\begin{array}{l}\text { GfA2364 (\%) } \\
648-R-183^{c}\left(0^{*}\right)\end{array}$ & $\begin{array}{l}\text { A.E.D., R.D.P., and R.H.P., unpublished results } \\
21\end{array}$ \\
\hline $57-7-7$ & none & Fum1-2, Fum2-1 & meiotic progeny & $\begin{array}{l}M-5500^{c} \text { ( ( ) } \\
M-3125 \text { (ठ) }\end{array}$ & 13 \\
\hline GfA2364 ${ }^{d}$ & none & Fum2-1 & FUM1 disruption mutant & $\mathrm{N} / \mathrm{A}$ & 20 \\
\hline
\end{tabular}

${ }^{a}$ Fumonisin production phenotypes and genotypes were determined previously by LC-MS and meiotic analyses (13, 21). Fum1-1 and Fum2-1 are functional alleles. Fum1-2 and Fum2-2 are nonfunctional alleles that originated in strains M-5500 and A-0822, respectively (see footnote $d$ for more details on these two strains) (11, 13). ${ }^{b}$ nd indicates not determined, and N/A indicates not applicable. ${ }^{c}$ Strain M-3120 was a field isolate from California, had the Fum1-1/Fum2-1 genotype, and produced the wild-type complement of $\mathrm{FB}_{1}, \mathrm{FB}_{2}, \mathrm{FB}_{3}$, and $\mathrm{FB}_{4}(35)$. Strain $\mathrm{A}-0822$ is a field isolate from South Carolina, had the Fum1-1/Fum2-2 genotype, and produced $\mathrm{FB}_{2}$ and $\mathrm{FB}_{4}$ only (11). Strain 648-R-193 was a meiotic progeny derived from strain A-0822, had the Fum1-1/Fum2-2 genotype, and produced FB 2 and FB 4 only (13, 21). Strain M-5500 was a field isolate from Nepal, had the Fum1-2/Fum2-1 genotype, and produced no fumonisins. ${ }^{d}$ Strain GfA2364 was generated from wild-type strain M-3125 by disruption of the FUM1 gene (20). The Fum1 genotype for strain GfA2364 is not included here because, on the basis of the results of the current study, it has neither the functional Fum1-1 allele nor the nonfunctional Fum1-2 allele.

was deficient in $\mathrm{C}-10$ hydroxylation and, as a result, produced only $\mathrm{FB}_{2}$ and $\mathrm{FB}_{4}$. Meiotic analyses indicated that these altered production phenotypes were caused by nonfunctional alleles at three closely linked loci. The nonproduction phenotype resulted from a nonfunctional allele at the Fuml locus (12), the C-10 hydroxylation deficiency resulted from a nonfunctional allele at the Fum2 locus, and the C-5 hydroxylation deficiency resulted from a nonfunctional allele at the Fum3 locus (13).

Molecular genetic analysis of $F$. verticillioides has identified a fumonisin biosynthetic gene (FUM) cluster that consists of 15 coregulated genes, all of which exhibit a pattern of expression that is correlated with fumonisin production (14). The roles of some of the clustered FUM genes in fumonisin biosynthesis have been determined (15-19). Several studies have provided indirect evidence for relationships between natural variations in fumonisin production and the clustered FUM genes. Three lines of evidence suggest that the naturally occurring fumonisinnonproduction phenotype may result from a mutation in or near the $F U M$ cluster. First, fumonisin-nonproducing strains of $F$. verticillioides can be generated by disruption (inactivation) of three FUM genes, the polyketide synthase gene FUM1 (previously FUM5), the cytochrome P450 monooxygenase gene FUM6, and the oxoamine synthase gene FUM8 $(18,20)$. Second, meiotic analysis indicated that the naturally occurring mutation that caused the fumonisin-nonproduction phenotype was loosely linked to FUM1 and therefore the FUM cluster (A.E.D., R.D.P., and R.H.P., unpublished results). Finally, introduction of cosmid clone 16-1, which had the entire FUM1 gene, only part of FUM6, and no other known FUM genes, restored fumonisin production in strain 57-7-7, which has the naturally occurring nonproduction phenotype that originated in Nepal (20). In contrast, cosmid clone 2-2, which had only part of FUM1 and no other known FUM genes, did not restore production to strain 57-7-7 (20). Although the latter results suggest that a mutation within FUM1 is responsible for the naturally occurring nonproduction phenotype, they are inconclusive. Because cosmid clone 16-1 was only partially characterized, the results do not rule out the possibility that some other gene(s) in clone 16-1 restored fumonisin production. There is even less evidence for an association between the C-10 hydroxylation deficiency and genes in the FUM cluster. Neither cosmid clone 2-2 nor 16-1 restored production of the wild-type complement of $\mathrm{FB}_{1}, \mathrm{FB}_{2}, \mathrm{FB}_{3}$, and $\mathrm{FB}_{4}$ when introduced into a strain with the naturally occurring $\mathrm{C}$ - 10 hydroxylation deficiency (20). Thus, the relationships of the fumonisin nonproduction and $\mathrm{C}-10$ hydroxylation-deficient phenotypes to the FUM gene cluster remain unresolved.

There is strong, albeit indirect, evidence for an association between the naturally occurring C-5 hydroxylation deficiency and the FUM cluster. The evidence was obtained from two laboratory-induced mutant strains of $F$. verticillioides that have the C-5 hydroxylation deficiency. The first mutant was generated by UV light mutagenesis (21), and the second was generated by specific deletion of a dioxygenase gene (FUM3) in the FUM cluster (22). Sequence analysis of the UV-light-induced mutant revealed a mutation in FUM3 that was predicted to render the gene nonfunctional. Because both laboratory-generated mutations of FUM3 resulted in the C-5 hydroxylation deficiency, it is likely that naturally occurring C-5 hydroxylation deficient strains also have a defect in FUM3.

The objective of this study was to elucidate the molecular genetic basis of the fumonisin-nonproduction and C-10 hydroxylation-deficient phenotypes that occur in natural variants of $F$. verticillioides. The results of this study will contribute to understanding fumonisin biosynthesis and the significance of different fumonisin chemotypes in the ecology of $F$. verticillioides and in maize agriculture. Some of the results of the current study have been previously reported in an abstract (23).

\section{MATERIALS AND METHODS}

Fungal Strains and Media. Previously identified strains of $F$. verticillioides used in this study and their fumonisin-production phenotypes are listed in Table 1. Strain ISU-93-152 was provided by Professor Donald White (University of Illinois). For production of conidia, strains were grown on V-8 juice agar medium (24) for 7-10 days, and for DNA preparation, strains were grown in liquid GYEP medium $(0.3 \%$ glucose, $0.1 \%$ yeast extract, $0.1 \%$ peptone) for $2-3$ days.

Nucleic Acid Manipulations. F. verticillioides genomic DNA was prepared from dried mycelia with the DNeasy Plant Mini Kit as described by the manufacturer (Qiagen, Valencia, CA). All DNA plasmids used as transformation vectors in deletion or complementation experiments were purified from Escherichia coli cells with the Midi Prep Kit (Qiagen). All Fusarium-derived DNA fragments that were amplified by Polymerase Chain Reaction (PCR) and used in the construction of vectors were amplified with PfuUltra (Stratagene, La Jolla, CA) or $P f x$ (Invitrogen Life Technologies, Carlsbad, CA) DNA polymerase. The nucleotide sequences of such fragments were determined to confirm that no errors were introduced during PCR. Sequencing reactions were done with the BigDye Terminator Cycle Sequencing (version 3.1, Applied Biosystems, Foster City, CA) 
protocol. Sequencing reactions were passed through a Sephadex G-50 column (Amersham Biosciences $\mathrm{AB}$, Piscataway, NJ), dried in a vacuum centrifuge, and resuspended in HiDi (Applied Biosystems) before electrophoretic analysis on a 3100 Genetic Analyzer (Applied Biosystems). Transformants of $F$. verticillioides were examined by both PCR and Southern blotting according to standard methods (25). Labeling of hybridization probes for Southern blots was done with the Prime-a-Gene system (Promega, Madison, WI) or Ready-to-Go (Amersham Biosciences $\mathrm{AB}$ ) protocol.

Vector Construction, Transformation, and Meiotic Crosses. The FUM12 deletion vector, p $\triangle F U M 12-H y g$, was constructed using the same approach previously described to construct the FUM3 and FUM13 deletion vectors $(16,22)$. The $1.4-\mathrm{kb}$ regions immediately up- and downstream of the FUM12 coding region were amplified and cloned into pT7Blue (Invitrogen Life Technologies). The primers used to amplify the upstream fragment were 5'-CGTGGATCCTGCCAGAAGAATGCCGAACCT-3' (underlined sequence is a BamHI site) and 5'-CATGGCGCGCCAAGAAGGGAGGGACTCGAGTCT-3' (underlined sequence is an $A s c \mathrm{I}$ site), and the primers used to amplify the downstream fragment were 5'-CATGGCGCGCCAAGCACAGGTTGAGTGGGATTG-3' (underlined sequence is an $A s c$ I site) and 5'GTCGAGCTCAGCACAGCATAGCCCACATGTC-3' (underlined sequence is a $\mathrm{Sa}$ I site). The two amplicons were cloned into the same vector (pT7Blue) with the BamHI, SacI, and AscI sites that were introduced via the PCR primers and/or present in the pT7Blue multiple cloning sequence. This cloning step restored the orientation and position of the two fragments found in the $F$. verticillioides genome except that the two fragments were separated by an AscI site instead of the FUM12 coding region. The $H y g B$ gene was then inserted between the upstream and downstream fragments via the AscI site to yield $\mathrm{p} \Delta \mathrm{FUM} 12-\mathrm{Hyg}$. Construction of the FUM3 (= FUM9) deletion vector, pFUM9KOH, was described previously (22).

The FUM12 complementation vector, pCFUM12-Hyg, was prepared by PCR amplification of a fragment spanning from 1052 bp upstream of the FUM12 start codon to 680 bp downstream of the FUM12 stop codon. The 3460-bp amplicon was cloned into the pCR XL TOPO vector (Invitrogen Life Technologies). A 2489-bp NotI fragment carrying the hygromycin resistance gene $H y g B$ was introduced into the resulting vector with the NotI site in the polylinker region of pCR XL TOPO. $H y g B$ was modified from pUCH2-8 $(26,27)$ by introduction of NotI sites at both ends of the gene by PCR amplification with primers 5'-CGATGCGGCCGCACCGGCTGCACATGTCAAGG-3' and 5'GCATGCGGCCGCACAGTTAAATTGCTAACGCAGTC-3' (NotI sites are underlined).

The FUM1 complementation vector was constructed from two overlapping DNA fragments obtained by restriction enzyme digestion of cosmid clone 16-1 $(14,20)$. The first fragment was obtained by DraI digestion of clone 16-1 and spanned from 29 bp upstream of the FUM1 start codon to 753 bp downstream of the FUM1 stop codon. The second fragment was obtained by SpeI digestion of clone 16-1 and spanned from $1473 \mathrm{bp}$ upstream to $1580 \mathrm{bp}$ downstream of the FUM1 start codon. The DraI fragment was cloned into pBluescript II (Stratagene), and the resulting construct was cut with SpeI and then ligated to the SpeI fragment described above. The resulting construct, pCFUM1, consisted of the entire FUM1 coding region plus 1473 bp of 5'-flanking and $753 \mathrm{bp}$ of 3 '-flanking DNA cloned in pBlueScript II. This plasmid was introduced into $F$. verticillioides by cotransformation with plasmid pUCH2-8 as previously described (28). pUCH2-8 carries the hygromycin phosphotransferase gene $\mathrm{HygB}$, which facilitates selection of transformants by their ability to grow on the antibiotic hygromycin $(26,27)$.

Transformation of $F$. verticillioides was done with the protoplast method described previously (20). Transformants were selected by their ability to grow on hygromycin B at $150 \mu \mathrm{g} / \mathrm{mL}$ (20). Sexual crosses of $F$. verticillioides were done with the carrot agar method described previously $(13,29)$.

Fumonisin Analysis. Strains were grown in 10-g cracked maize kernel cultures and or in 20-mL GYAM medium cultures as previously described (18). After a 3-week incubation, cracked maize kernel cultures were extracted with $5 \mathrm{~mL}$ of acetonitrile/water $(1: 1, \mathrm{v} / \mathrm{v})$ per gram of culture. After a 2-week incubation, GYAM cultures were filtered through $0.2-\mu \mathrm{m}$ Nalgene filters. Extracts and filtrates were analyzed by HPLC-mass spectrometry (MS) for the presence of fumonisins (30). The HPLC system employed an Intersil ODS3 column $(10 \mathrm{~cm}, 5 \mu \mathrm{m})$, a flow rate of $0.3 \mathrm{~mL} \mathrm{~min}^{-1}$, and a gradient solvent system that began with water/methanol/acetic acid (65:35:0.35) and changed to water/ methanol/acetic acid (5:95:0.35) over $10 \mathrm{~min}$. The solvent was maintained at the latter ratio for $15 \mathrm{~min}$ and then returned to the former ratio over a period of $1 \mathrm{~min}$. The HPLC column was coupled to an API Source of a Finnigan LCQ Deca MS System (ThermoQuest, San Jose, CA) operated in the electrospray (ESI) mode. The MS interface capillary temperature was $255^{\circ} \mathrm{C}$, and the spray voltage was $4.5 \mathrm{kV}$. The MS scanned for ions from 250 to 950 mass units. $\mathrm{FB}_{1}, \mathrm{FB}_{2}, \mathrm{FB}_{3}$, and $\mathrm{FB}_{4}$ were identified by retention time in comparison to standards and by their ESI spectra.

\section{RESULTS}

Identification of the Fumonisin C-10 Hydroxylase Gene. The FUM12 gene is located in the FUM gene cluster and encodes a cytochrome P450 monooxygenase (14). To determine whether this gene is required for fumonisin biosynthesis, we deleted the FUM12 coding region in wild-type $F$. verticillioides strain M-3125 via transformation with deletion vector $\mathrm{p} \Delta$ FUM12Hyg. The vector was designed so that homologous recombination between FUM12 sequences in pDFUM12-Hyg and in the $F$. verticillioides genome would replace the FUM12 coding region with the $H y g B$ gene and thereby delete the coding region. One hundred and twenty-two hygromycin-resistant putative transformants were analyzed by PCR, and only two (strains GfA2874 and GmT201) yielded the amplification products expected for deletion of the FUM12 coding region and its replacement with $H y g B$. The deletion was confirmed by Southern blot analysis in which genomic DNA was digested with restriction endonuclease $S n a B I$ and, after electrophoresis and blotting, hybridized to a probe that spanned from $1032 \mathrm{bp}$ upstream to 933 bp downstream of the FUM12 start codon. The probe hybridized to a 2.9-kb DNA fragment in wild-type strain M-3125 but to a 3.7-kb fragment in the FUM12 deletion mutants GfA2874 and GmT201 (Figure 2A). On the basis of known sequence data, the expected size of the SnaBI fragment with FUM12 in wild-type $F$. verticillioides is $2.9 \mathrm{~kb}$, and the expected size of the SnaBI fragment in which the FUM12 coding region is replaced by the $H y g B$ gene is $3.7 \mathrm{~kb}$.

LC-MS analysis of cracked maize culture extracts revealed that the two FUM12 deletion mutants produced only $\mathrm{FB}_{2}$ and $\mathrm{FB}_{4}$, both of which lack the C-10 hydroxyl. In contrast, the progenitor strain M-3125 and transformants in which FUM12 was not deleted produced the wild-type complement of fumonisins including the $\mathrm{C}$-10-hydroxylated $\mathrm{FB}_{1}$ and $\mathrm{FB}_{3}$. In this experiment, the levels of fumonisins produced in two cultures of M-3125 were 5300 and $12200 \mu \mathrm{g}$ of $\mathrm{FB}_{1}, 1900$ and $3700 \mu \mathrm{g}$ of $\mathrm{FB}_{2}$, and 900 and $1300 \mu \mathrm{g}$ of $\mathrm{FB}_{3}$ per gram of cracked maize culture. The level of $\mathrm{FB}_{2}$ produced by the two FUM12 disruption mutants was 2400 (GfA2874) to 9200 (GmT201) $\mu$ g per gram of cracked maize culture. The presence of $\mathrm{FB}_{4}$ was noted but not quantified in this experiment. Given that cytochrome P450 monooxygenases often catalyze hydroxylation reactions, these results indicate that the FUM12-encoded monooxygenase catalyzes fumonisin $\mathrm{C}-10$ hydroxylation.

Complementation of the Naturally Occurring C-10 Hydroxylation Deficiency. The inability of FUM12 deletion mutants to hydroxylate the fumonisin backbone at $\mathrm{C}-10$ suggests that strains with the naturally occurring C-10 hydroxylation deficiency may have a mutation within FUM12. To address this possibility, we used transformation to introduce a wild-type copy of FUM12 into two strains, 109-R-14 and 982-R-50, with the 


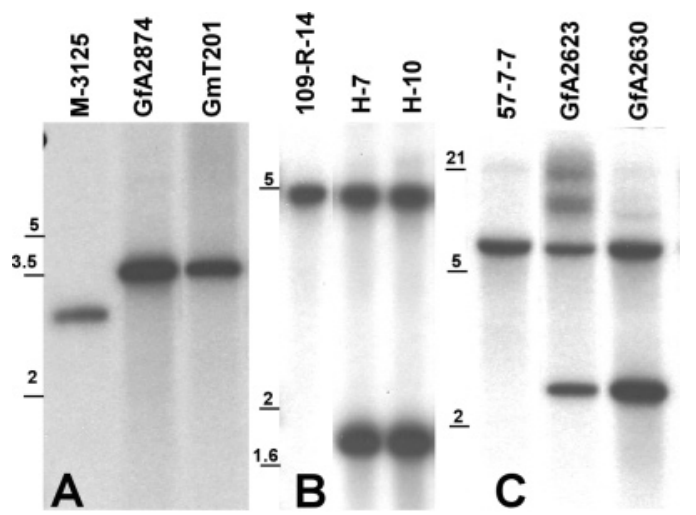

Figure 2. Southern blots of complementation and deletion strains. (A) Deletion of FUM12 coding region. Lanes: M-3125, wild-type strain; GfA2874 and GmT201, FUM12 deletion mutants. Under the conditions of this analysis, the wild-type strain was expected to yield a $2.9-\mathrm{kb}$ band and strains in which the FUM12 coding region was replaced by the $H y g B$ gene were expected to yield a $3.7-\mathrm{kb}$ band. (B) Complementation of the C-10 hydroxylation-deficient phenotype by transformation with the wildtype FUM12 gene in pCFUM12-Hyg. Lanes: 109-R-14, C-10 hydroxylation-deficient progenitor strain; $\mathrm{H} 7$ and $\mathrm{H} 10$, strains generated by transformation of strain 109-R-14 with pCFUM12-Hyg. Under the conditions of this analysis, the endogenous FUM12 gene was expected to be present as a 4.6-kb band and the FUM12 in vector pFUM12-Hyg was expected to be present on a $1.7-\mathrm{kb}$ band. (C) Complementation of the fumonisinnonproducing phenotype by transformation with the wild-type FUM1 gene in vector pCFUM1. Lanes: 57-7-7, strain with naturally occurring nonproduction phenotype; GfA2623 and GfA2630, strains generated by transformation of 57-7-7 with pCFUM1. Under the conditions of this analysis, the endogenous FUM1 from strain 57-7-7 was expected yield a $6.9-\mathrm{kb}$ band and the FUM1 from vector pCFUM1 was expected to yield a 2.7-kb band. Within panels A-C, each lane is from the same blot, but lanes between those shown have been removed. Numbers to the left of each panel indicate the positions of selected molecular weight markers (kb) generated by digestion of phage $\lambda$ DNA with $E c 0 R I$ and Hindlll.

naturally occurring $\mathrm{C}-10$ hydroxylation deficiency. These two strains are meiotic progeny derived from the original C-10 hydroxylation-deficient field isolate A-0822, which was isolated from maize grown in South Carolina (Table 1). The meiotic progeny were used in this experiment instead of the original field isolate because they were more amenable to genetic analysis. The wild-type copy of FUM12 was present in complementation vector pCFUM12-Hyg, and transformants were selected by their ability to grow on hygromycin B-amended media.

LC-MS analysis of GYAM culture filtrates revealed that 9 of 11 109-R-14 transformants and 4 of 7 982-R-50 transformants produced the wild-type complement of $\mathrm{FB}_{1}, \mathrm{FB}_{2}, \mathrm{FB}_{3}$, and $\mathrm{FB}_{4}$. In contrast, strains $109-\mathrm{R}-14$ and $982-\mathrm{R}-50$ and the remaining transformants produced only $\mathrm{FB}_{2}$ and $\mathrm{FB}_{4}$. In this experiment, the 15 complemented transformants of fumonisin production ranged from 7 to $312 \mu \mathrm{g}$ of $\mathrm{FB}_{1}$, from 1 to $43 \mu \mathrm{g}$ of $\mathrm{FB}_{2}$, from 1 to $49 \mu \mathrm{g}$ of $\mathrm{FB}_{3}$, and from $<1$ to $6 \mu \mathrm{g}$ of $\mathrm{FB}_{4}$ per milliliter of GYAM medium. Fumonisin production by the two progenitor strains 109-R-14 and 982-R-50 ranged from 73 to $84 \mu \mathrm{g}$ of $\mathrm{FB}_{2}$ and from 2 to $6 \mu \mathrm{g}$ of $\mathrm{FB}_{4}$ per milliliter of GYAM medium.

The presence of the complementation vector in transformants was confirmed by Southern blot analysis (Figure 2B). In this analysis, genomic DNA was digested with EcoRI, electrophoresed, blotted, and hybridized to a ${ }^{32} \mathrm{P}$-labeled probe corresponding to nucleotides $816-1593$ of the FUM12 coding region. On the basis of known sequence data, the region of the endogenous FUM12 complementary to the hybridization was predicted to be present as a 4.6-kb EcoRI fragment, whereas the same region in the complementation vector pCFUM12-Hyg was predicted to be present as a 1.7-kb EcoRI fragment. In the Southern analysis, strains 109-R-14 and 982-R-50 had the 4.6$\mathrm{kb}$ fragment only, but all transformants with wild-type fumonisin production had both the 4.6- and 1.7-kb fragments (Figure 2B). Thus, introduction of a wild-type FUM12 gene into strains with the naturally occurring C-10 hydroxylation deficiency could restore wild-type fumonisin production.

FUM12 Sequence in a C-10 Hydroxylation-Deficient Strain. Complementation of the naturally occurring C-10 hydroxylation deficiency via transformation with a wild-type FUM12 indicates that there is a mutation within FUM12 in the deficient strains. To address this possibility, the entire FUM12 coding region was amplified by PCR from strain 109-R-14 and then sequenced. The sequence analysis revealed six nucleotide differences in the amplified FUM12 coding region compared to the previously described wild-type FUM12 sequence (GenBank accession AF155773). All differences were confirmed in two independently amplified PCR products. Five of the differences were nucleotide substitutions; two of these altered and three did not alter the amino acid specificity of the codons in which they occurred. The sixth difference was a $\mathrm{T}$ insertion between nucleotides 72 and 73 of the FUM12 coding region. The insertion caused a frame shift that introduced multiple stop codons into the FUM12 coding region. Such a frame shift would cause incorrect translation as well as truncation of the FUM12encoded cytochrome P450 monooxygenase. Thus, the frame shift mutation would almost certainly render FUM12 nonfunctional.

Generation of C-5 and C-10 Hydroxylation-Deficient Strain. If the FUM12-encoded P450 monooxygenase catalyzes fumonisin C-10 hydroxylation and, as previously demonstrated, the FUM3-encoded dioxygenase catalyzes C-5 hydroxylation $(17,22)$, a double mutant in which both FUM12 and FUM3 are nonfunctional should be deficient in both C-5 and C-10 hydroxylation. To test this hypothesis, the FUM3 gene was deleted in the C-10 hydroxylation-deficient strain 982-R-50. Construction of the FUM3 deletion vector and analysis of transformants were done as described previously for FUM3 deletion in wild-type strain M-3125 (22). PCR analysis indicated that FUM3 was deleted in only 1 of 52 transformants examined. LC-MS analysis of cracked maize extracts of this FUM3 deletion mutant revealed that it produced only $\mathrm{FB}_{4}$, which lacks both the C-5 and C-10 hydroxyls (Figure 3). As far as we are aware, this fumonisin production phenotype has not been described previously. In this experiment, the progenitor strain 982-R-50 produced $38 \mu \mathrm{g}$ of $\mathrm{FB}_{2}$ and $11 \mu \mathrm{g}$ of $\mathrm{FB}_{4}$ per milliliter of GYAM and the FUM3 deletion mutant (in the genetic background of strain 982-R-50) produced $21 \mu \mathrm{g}$ of $\mathrm{FB}_{4}$ per milliliter of GYAM medium.

Characterization of a Naturally Occurring FumonisinNonproducing Variant. LC-MS analysis of cracked maize cultures of $F$. verticillioides field isolate ISU-93-152 (Table 1) revealed that it did not produce detectable levels of fumonisins. In the same experiments, wild-type strain M-3125 produced approximately $8000 \mu \mathrm{g}$ of $\mathrm{FB}_{1}, \mathrm{FB}_{2}$, and $\mathrm{FB}_{3}$ combined per gram of cracked maize culture. To examine the inheritance of the fumonisin-nonproducing phenotype, strain ISU-93-152 was sexually crossed to wild-type strain M-3125. Of 20 singleascospore progeny examined, 6 produced the wild-type complement of $\mathrm{FB}_{1}, \mathrm{FB}_{2}$, and $\mathrm{FB}_{3}$ and 14 did not produce fumonisins. Strain ISU-93-152 was also crossed with strain GfA2364, a 


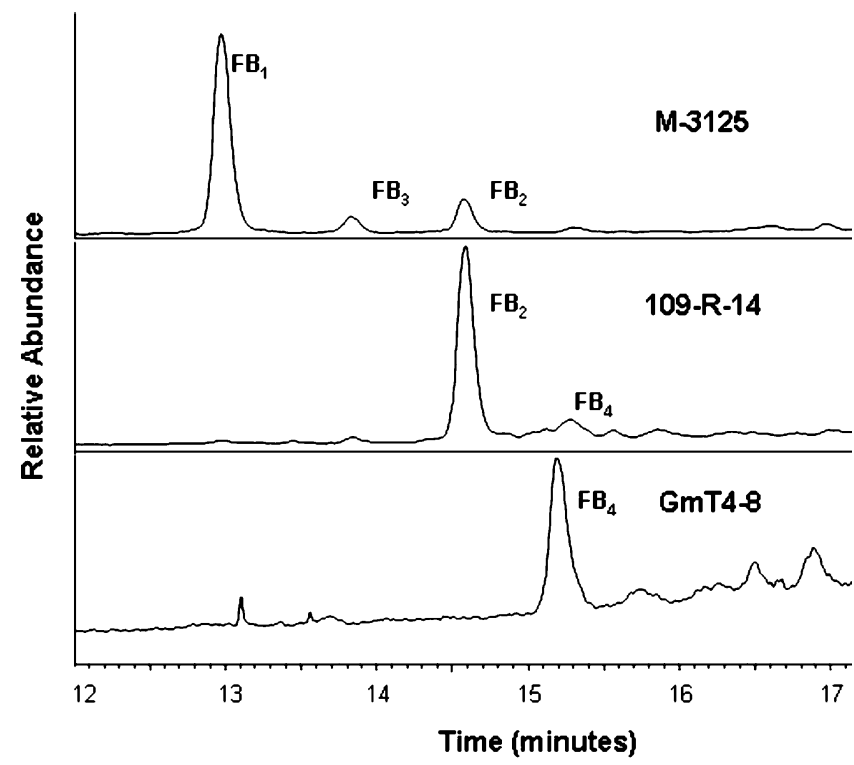

Figure 3. Total ion chromatograms from LC-MS analysis of GYAM culture filtrates of wild-type strain M-3125, C-10 hydroxylation deficient strain 982R-50, and strain GmT4-8, which was generated by deletion of FUM3 in strain $982-R-50$.

fumonisin-nonproducing mutant generated by disruption of the polyketide synthase gene, $F U M 1$, in the fumonisin biosynthetic gene cluster (14). All 19 progeny examined from this second cross exhibited the same fumonisin-nonproduction phenotype of the two parental strains. Therefore, no recombination between the mutation in ISU-93-152 and the disrupted FUM1 gene was evident. Although these genetic analyses included only limited numbers of progeny, they are consistent with the fumonisinnonproduction phenotype of strain ISU-93-152 being a heritable trait that results from a mutation(s) that is (are) at least loosely linked to the FUMI gene and therefore the FUM cluster.

The loose linkage to the FUM gene cluster raises the possibility that the fumonisin-nonproduction phenotype of strain ISU-93-152 results from a mutation within the cluster. To address this possibility, ISU-93-152 was transformed independently with two overlapping cosmid clones (6B and 4-5), each of which carried an incomplete set of cluster genes. Cosmid clone 6B carried a complete copy of FUM1, FUM6, and FUM7 but no other cluster genes, and cosmid clone 4-5 carried all of the cluster genes (FUM3, FUM7, FUM8, and FUM10-FUM19) except for FUM1 and FUM6 (14). Five hygromycin-resistant isolates recovered following transformation with each cosmid clone were analyzed for fumonisin production. Four isolates recovered following transformation with clone $6 \mathrm{~B}$ were restored to fumonisin production, but none of the isolates recovered following transformation with clone 4-5 were restored to fumonisin production. These results indicate that clone $6 \mathrm{~B}$ has a wild-type copy of the gene that is mutated in strain ISU-93152 and that clone 4-5 does not. The fact that disruption of FUM1 and FUM6 blocks fumonisin production (18) combined with the presence of these two genes in clone 6B suggests that the nonproduction phenotype of strain ISU-93-152 results from a mutation in one of them.

Complementation of Fumonisin-Nonproduction Phenotype with $F \boldsymbol{U M 1}$. If the naturally occurring fumonisin-nonproduction phenotype results from a mutation within $F U M 1$ or FUM6, introduction of a wild-type copy of these two genes into a nonproducing strain should restore fumonisin production. To determine whether nonproduction can be restored by FUM1, we used transformation to introduce a wild-type copy of FUMI into two strains (ISU-93-152 and 57-7-7) with the naturally occurring nonproduction phenotype. Strain ISU-93-152 is a field isolate obtained from maize grown in Iowa (31), and strain 577-7 is a progeny of the fumonisin-nonproducing field isolate, M-5500, from Nepal (Table 1). We used strain 57-7-7 rather than M-5500 because it was more amenable to genetic analysis and had been used successfully in previous transformation studies (20).

The wild-type FUM1 used in this study was present in complementation vector pCFUM1, which was cotransformed (28) into strains ISU-93-152 and 57-7-7. Transformants were selected by their ability to grow on hygromycin B-amended media. LC-MS analysis of cracked maize culture extracts of 19 hygromycin-resistant transformants recovered following transformation of strain 57-7-7 with pCFUM1 revealed that 15 produced the wild-type complement of $\mathrm{FB}_{1}, \mathrm{FB}_{2}$, and $\mathrm{FB}_{3}$ (the presence of $\mathrm{FB}_{4}$ was not determined). The remaining four transformants had the fumonisin-nonproduction phenotype of progenitor strain 57-7-7. Likewise, LC-MS analysis of culture extracts of 23 isolates recovered following transformation of strain ISU-93-152 revealed that two produced the wild-type complement of fumonisins. The levels of fumonisins produced by the 17 complemented transformants of 57-7-7 and ISU-93152 ranged from 240 to $4500 \mu \mathrm{g}$ of $\mathrm{FB}_{1}$, from 21 to $1463 \mu \mathrm{g}$ of $\mathrm{FB}_{2}$, and from 62 to $3067 \mu \mathrm{g}$ of $\mathrm{FB}_{3}$ per gram of cracked maize culture ( $\mathrm{FB}_{4}$ was detected but not quantified in this experiment). Southern blot analysis of a subset of transformants derived from both nonproducing progenitor strains confirmed the presence of the complementation vector in fumonisin-producing transformants (Figure 2C). For this analysis, genomic DNA was digested with $\mathrm{XbaI}$ and the hybridization probe spanned from 503 bp upstream to 1051 bp down stream of the FUM1 translation start site. On the basis of known sequence data, the region of the endogenous FUM1 complementary to the hybridization probe should have been present as a 6.9-kb XbaI fragment, whereas the same region in vector pCFUM1 should have been present as a 2.7-kb XbaI fragment. The Southern blot analysis revealed that all fumonisin-producing transformants had both the 6.9- and 2.7-kb fragments. The progenitor strains and selected nonproducing transformants had the $6.9-\mathrm{kb}$ fragment only. These results indicate that the naturally occurring fumonisin-nonproduction phenotype in strains 57-7-7 and ISU-93152 could be complemented by introduction of a wild-type copy of FUM1.

FUM1 Sequence in a Fumonisin-Nonproducing Strain. Complementation of two strains with the naturally occurring fumonisin-nonproduction phenotype via introduction of a wildtype copy of FUM1 suggests that these strains have a mutation within their FUM1 gene that renders the gene nonfunctional. To address this possibility, we amplified and sequenced the entire 8.16-kb FUM1 coding region from fumonisin-nonproducing strain 57-7-7. The sequence analysis revealed 20 nucleotide differences in the FUM1 coding region of strain 577-7 compared to the previously reported wild-type FUMI sequence (GenBank accession AF155773). All differences consisted of single nucleotide substitutions, 12 of which affected and 7 of which did not affect the amino acid specificity of the codons in which they occurred. Only one of the nucleotide substitutions, a G-to-A transition at nucleotide 5415 of the protein coding region, disrupted FUM1 by introducing a premature stop codon that would stop translation prior to synthesis of the enoyl reducatase, keto-reductase, and acyl carrier domains of the polyketide synthase encoded by FUM1 (20). Thus, the presence of the G-to-A transition at nucleotide 5415 
would almost certainly render FUM1 nonfunctional. The presence of the G-to-A transition was confirmed by sequence analysis of a 700-bp amplicon that spanned nucleotide 5415 .

Sequence analysis of the same 700-bp region of FUM1 in strain ISU-93-152 revealed that this strain did not differ from the wild type at nucleotide 5415. In addition, overlapping 1.5-2 kb DNA fragments amplified by PCR from along the entire length of the FUM1 coding region in strain ISU-93-152 and wild-type strain did not exhibit a noticeable difference in size when viewed on an ethidium bromide-stained agarose gel (data not shown). These data indicate that the mutation in the ISU93-152 FUM1 gene responsible for the nonproduction phenotype is not a large ( $>50 \mathrm{bp}$ ) insertion or deletion in the coding region.

\section{DISCUSSION}

Natural variation in mycotoxin production within individual fungal species and between closely related species has received considerable attention because of the potential impact of such variation on human and animal health, on international trade, on biological control of mycotoxin contamination, and on understanding mycotoxin biosynthesis. In the cases of aflatoxins and trichothecenes, the molecular genetic basis of some of the variation has been demonstrated. For example, some field isolates of Aspergillus flavus do not produce aflatoxins because of a single nucleotide substitution that introduces a premature stop codon in the coding region of the polyketide synthase gene in the aflatoxin biosynthetic gene cluster (32). In field isolates of $F$. graminearum, production of the trichothecenes nivalenol and 4-acetylnivalenol versus deoxynivalenol can result from multiple deletions and insertions in a monooxygenase gene (Tri13) and an acyltransferase gene (Tri7) in the trichothecene biosynthetic gene cluster $(33,34)$. Prior to the current study, characterization of the molecular genetic basis of natural variation in fumonisin production in $F$. verticillioides has been incomplete. The results reported here, however, provide direct evidence that this variation can result from point mutations in genes in the fumonisin biosynthetic gene cluster.

Three lines of evidence from the current study indicate that the $\mathrm{C}-10$ hydroxylation-deficient phenotype resulted from a mutation within the FUM12 gene. First, laboratory-induced FUM12 deletion mutants exhibited the C-10 hydroxylation deficiency; second, the naturally occurring deficiency was complemented by transformation with a wild-type copy of FUM12; and third, the FUM12 coding sequence in a strain with the naturally occurring deficiency had a frame shift mutation predicted to render the FUM12-encoded monooxygenase nonfunctional. Previous meiotic analyses determined that the naturally occurring $\mathrm{C}-10$ hydroxylation deficiency was caused by a nonfunctional allele at the Fum2 locus (13). Thus, the data reported in the current study also indicate that the meiotically defined Fum 2 locus and the molecularly defined FUM12 are the same gene. Because Fum2 was described prior to FUM12, we propose that hereafter the gene be designated FUM2. Furthermore, because the results of this study revealed that the monooxygenase encoded by FUM2 is responsible for fumonisin C-10 hydroxylation, they further define the functions of the FUM cluster in $F$. verticillioides.

Complementation experiments in the current study also localized the naturally occurring fumonisin nonproduction phenotype in two strains with distant geographic origins to the polyketide synthase gene, $F U M 1$, in the $F U M$ cluster. Furthermore, sequence analysis indicated that the nonproduction phenotype in strain 57-7-7 most likely resulted from a nucleotide substitution that introduced a premature stop codon in the FUMI coding region. Restoration of wild-type fumonisin production to strain ISU-93-152 by transformation with FUM1 indicated that the mutation responsible for the fumonisin-nonproduction phenotype in ISU-93-152 was located in FUM1. Limited sequence analysis demonstrated that the mutation was not located in the same position as in strain 57-7-7 and did not resolve whether the mutation in ISU-93-152 was in the coding, promoter, or termination region of FUM1. Previously reported meiotic analyses demonstrated that the fumonisin-nonproduction phenotype in 57-7-7 resulted from a nonfunctional allele at the Fuml locus $(13,21)$. Thus, the results from the current study combined with the previous meiotic analysis indicate that the molecularly defined FUMI gene and the meiotically defined Fuml locus are the same gene. In keeping with the current designation of $F U M$ and other $F$. verticillioides genes, we propose that hereafter the gene be referred to as FUMI.

When compared to the published sequences for wild-type $F$. verticillioides, FUM1 in strain 57-7-7 and FUM2 (= FUM12) in strain 109-R-14 included nucleotide substitutions in addition to those that introduced the premature stop codon in FUM1 and frame shift in FUM2. Some of these other changes affected the amino acid specificity of the codons in which they occurred. However, it is not clear whether the resulting amino acid substitutions would affect the activity of the FUM1 or FUM2 enzymes. Regardless, the premature stop codon in FUM1 of 57-7-7 and the frame shift in FUM2 of 109-R-14 would cause such profound changes in the corresponding proteins that the proteins would be nonfunctional.

Most field isolates of $F$. verticillioides produce the full complement of $\mathrm{FB}_{1}, \mathrm{FB}_{2}, \mathrm{FB}_{3}$, and $\mathrm{FB}_{4}$ (11). The scarcity of the nonproduction and $\mathrm{C}-5$ and $\mathrm{C}-10$ hydroxylation-deficient phenotypes in natural populations of the fungus may be an indication that $\mathrm{FB}_{1}$ production contributes to the competitiveness of the fungus. Characterization of fumonisin biosynthetic genes and elucidation of the genetic basis of naturally occurring altered fumonisin production phenotypes should contribute to understanding the role, if any, of the toxins in the ecology of $F$. verticillioides.

\section{ACKNOWLEDGMENT}

We thank Marcie L. Moore, Deborah S. Shane, and Stephanie N. Folmar for technical assistance and Adrienne Kelly-Webb and Amy Morgan for synthesizing oligonucleotides and electrophoretic analysis of sequence reactions.

\section{LITERATURE CITED}

(1) Howard, P. C.; Eppley, R. M.; Stack, M. E.; Warbritton, A.; Voss, K. A.; Lorentzen, R. J.; Kovach, R. M.; Bucci, T. J. Fumonisin B1 carcinogenicity in a two-year feeding study using F344 rats and B6C3F1 mice. Environ. Health Perspect. 2001, 109 (S2), 277-282.

(2) Sadler, T. W.; Merrill, A. H.; Stevens, V. L.; Sullards, M. C.; Wang, E.; Wang, P. Prevention of fumonisin B1-induced neural tube defects by folic acid. Teratology 2002, 66, 169-176.

(3) Hendricks, K. Fumonisins and neural tube defects in south Texas. Epidemiology 1999, 10, 198-200.

(4) Marasas, W. F. O. Fumonisins: history, world-wide occurrence and impact. In Fumonisins in Food; Jackson, L. S., DeVaries, W., Bullerman, L. B., Eds.; Plenum Publishing: New York, 1996.

(5) Nelson, P. E.; Plattner, R. D.; Shackelford, D. D.; Desjardins, A. E. Fumonisin $\mathrm{B}_{1}$ production by Fusarium species other than F. moniliforme in Section Liseola and by some related species. Appl. Environ. Microbiol. 1992, 58, 984-989. 
(6) Rheeder, J. P.; Marasas, W. F. O.; Vismer, H. F. Production of fumonisin analogs by Fusarium species. Appl. Environ. Microbiol. 2002, 68, 2101-2105.

(7) Munkvold, G. P.; Desjardins, A. E. Fumonisins in maize: can we reduce their occurrence? Plant Dis. 1997, 81, 556-565.

(8) Bezuidenhout, S. C.; Gelderblom, W. C. A.; Gorst-Allman, C. P.; Horak, R. M.; Marasas, W. F. O.; Spiteller, G.; Bleggaar, R. Structure elucidation of the fumonisins, mycotoxins from Fusarium moniliforme. J. Chem. Soc., Chem. Commun. 1988, 1988, 743-745.

(9) Musser, S. M.; Plattner, R. D. Fumonisin composition in cultures of Fusarium moniliforme, Fusarium proliferatum, and Fusarium nygami. J. Agric. Food Chem. 1997, 45, 1169-1173.

(10) Desjardins, A. E.; Plattner, R. D.; Shackelford, D. D.; Leslie, J. F.; Nelson, P. E. Heritability of fumonisin $\mathrm{B}_{1}$ production in Gibberella fujikuroi mating population A. Appl. Environ. Microbiol. 1992, 58, 2799-2805.

(11) Plattner, R. D.; Desjardins, A. E.; Leslie, J. F.; Nelson, P. E. Identification and characterization of strains of Gibberella fujikuroi mating population A with rare fumonisin production phenotypes. Mycologia 1996, 88, 416-424.

(12) Desjardins, A. E.; Plattner, R. D.; Nelson, P. E.; Nelsen, T. C.; Leslie, J. F. Genetic analysis of fumonisin production and virulence of Gibberella fujikuroi mating population A (Fusarium moniliforme) on maize (Zea mays) seedlings. Appl. Environ. Microbiol. 1995, 61, 79-86.

(13) Desjardins, A. E.; Plattner, R. D.; Proctor, R. H. Linkage among genes responsible for fumonisin biosynthesis in Gibberella fujikuroi mating population A. Appl. Environ. Microbiol. 1996, 62, 2571-2576.

(14) Proctor, R. H.; Brown, D. W.; Plattner, R. D.; Desjardins, A. E. Co-expression of 15 contiguous genes delineates a fumonisin biosynthetic gene cluster in Gibberella moniliformis. Fungal Genet. Biol. 2003, 38, 237-249.

(15) Bojja, R. S.; Cerny, R. L.; Proctor, R. H.; Du, L. Determining the biosynthetic sequence in the early steps of the fumonisin pathway by use of three gene-disruption mutants of Fusarium verticillioides. J. Agric. Food Chem. 2004, 52, 2855-2860.

(16) Butchko, R. A. E.; Plattner, R. D.; Proctor, R. H. FUM13 encodes a short chain dehydrogenase/reductase required for C-3 carbonyl reduction during fumonisin biosynthesis in Gibberella moniliformis. J. Agric. Food Chem. 2003, 51, 3000-3006.

(17) Ding, Y.; Bojja, R. S.; Du, L. Fum3p, a 2-ketoglutarate-dependent dioxygenase required for C-5 hydroxylation of fumonisins in Fusarium verticillioides. Appl. Environ. Microbiol. 2004, 70, 1931-1934.

(18) Seo, J.-A.; Proctor, R. H.; Plattner, R. D. Characterization of four clustered and coregulated genes associated with fumonisin biosynthesis in Fusarium verticillioides. Fungal Genet. Biol. 2001, 34, 155-165.

(19) Yi, H.; Bojja, R. S.; Fu, J.; Du, L. Direct evidence for the function of FUM13 in 3-ketoreduction of mycotoxin fumonisins in Fusarium verticillioides. J. Agric. Food Chem. 2005, 53, 54565460.

(20) Proctor, R. H.; Desjardins, A. E.; Plattner, R. D.; Hohn, T. M. A polyketide synthase gene required for biosynthesis of fumonisin mycotoxins in Gibberella fujikuroi mating population A. Fungal Genet. Biol. 1999, 27, 100-112.

(21) Proctor, R. H.; Desjardins, A. E.; Plattner, R. D. Biosynthetic and genetic relationships of B-series fumonisins produced by Gibberella fujikuroi mating population A. Nat. Toxins 1999, 7 , 251-258.
(22) Butchko, R. A. E.; Plattner, R. D.; Proctor, R. H. FUM9 is required for $\mathrm{C}-5$ hydroxylation of fumonisins and complements the meiotically defined Fum3 locus in Gibberella moniliformis. Appl. Environ. Microbiol. 2003, 69, 6935-6937.

(23) Proctor, R. H.; Desjardins, A. E.; Plattner, R. D. Reconciling meiotically and molecularly defined fumonisin biosynthetic genes in Gibberella moniliformis. Phytopathology 2002, 92, S67.

(24) Tuite, J. Plant Pathological Methods: Fungi and Bacteria; Burgess Publishing: Minneapolis, MN, 1969.

(25) Sambrook, J.; Fritsch, E. F.; Maniatis, T. Molecular Cloning, A Laboratory Manual, 2nd ed.; Cold Spring Harbor Laboratory Press: Plainview, NY, 1989.

(26) Alexander, N. J.; Hohn, T. M.; McCormick, S. P. The TRI11 gene of Fusarium sporotrichioides encodes a cytochrome P450 monooxygenase required for $\mathrm{C}-15$ hydroxylation in trichothecene biosynthesis. Appl. Environ. Microbiol. 1998, 64, 221-225.

(27) Turgeon, B. G.; Garber, R. C.; Yoder, O. C. Development of a fungal transformation system based on selection of sequences with promoter activity. Mol. Cell. Biol. 1987, 7, 3297-3305.

(28) Mikkelsen, L.; Sarrocco, S.; Lubeck, S.; Jensen, D. F. Expression of the red fluorescent protein DsRed-Express in filamentous ascomycete fungi. FEMS Microbiol. Lett. 2003, 233, 135-139.

(29) Klittich, C. J. R.; Leslie, J. F. Nitrate reduction mutants of Fusarium moniliforme (Gibberella fujikuroi). Genetics 1988, 118 , 417-423.

(30) Plattner, R. D.; Weisleder, D.; Poling, S. M. Analytical determination of fumonisins and other metabolites produced by Fusarium moniliforme and related species on corn. In Fumonisins in Food; Jackson, L. S., DeVries, J. W., Bullerman, L. B., Eds.; Plenum Press: New York, 1996.

(31) Clements, M. J.; Maragos, C. M.; Pataky, J. K.; White, D. G. Sources of resistance to fumonisin accumulation in grain and Fusarium ear and kernel rot of corn. Phytopathology 2004, 94, 251-260.

(32) Ehrlich, K. C.; Cotty, P. J. An isolate of Aspergillus flavus used to reduce aflatoxin contamination in cottonseed has a defective polyketide synthase gene. Appl. Microbiol. Biotechnol. 2004, 65, 473-478.

(33) Brown, D. W.; McCormick, S. P.; Alexander, N. J.; Proctor, R. H.; Desjardins, A. E. Inactivation of a cytochrome P-450 is a determinant of trichothecene diversity in Fusarium species. Fungal Genet. Biol. 2002, 36, 224-233.

(34) Lee, T.; Han, Y.-K.; Kim, K.-H.; Yun, S.-H.; Lee, Y.-W. Tri13 and Tri7 determine deoxynivalenol- and nivalenol-producing chemotypes of Gibberella zeae. Appl. Environ. Microbiol. 2002, $68,2148-2154$.

(35) Leslie, J. F.; Plattner, R. D.; Desjardins, A. E.; Klittich, C. J. R. Fumonisin $\mathrm{B}_{1}$ production by strains from different mating populations of Gibberella fujikuroi (Fusarium section Liseola). Phytopathology 1992, 82, 341-345.

Received for review November 8,2005 . Revised manuscript received January 3, 2006. Accepted January 11, 2006. Names are necessary to report factually on available data; however, the USDA neither guarantees nor warrants the standard of the products, and the use of the name by the USDA implies no approval of the product to the exclusion of others that may also be suitable.

JF0527706 